\title{
Bifrequency pendulum on a rotary platform to teach optical birefringence and liquid crystalline displays
}

\section{Soileau, Boris Zeldovich}

M. J. Soileau, Boris Ya. Zeldovich, "Bifrequency pendulum on a rotary platform to teach optical birefringence and liquid crystalline displays," Proc. SPIE 2525, 1995 International Conference on Education in Optics, (13 October 1995); doi: 10.1117/12.224054

Event: SPIE's 1995 International Symposium on Optical Science, Engineering, and Instrumentation, 1995, San Diego, CA, United States 
Bifrequecy pendulum on a rotary platform to teach optical birefringence and liquid crystalline displays

M. J. Soileau, B. Ya. Zel'dovich

Center for Research and Education in Optics and Lasers, University of Central Florida, 12424 Research Parkway, Suite 400,

Orlando, FL, 32826, USA. Fax (407) 658-6880, e-addresses: mj@creol.ucf.edu, boris@creol.ucf.edu

\begin{abstract}
Bifrequency pendulum on a rotary platform was tested and used for several years as a tool to teach various optical phenomena, mostly connected with the polarization of light waves. The following optical topics have found their mechanical analogs: linearity and superposition; damping; isotropy versus anisotropy; birefringence; uniaxial crystals; adiabatic following versus abrupt changes; liquid crystalline displays for watches, based on a twisted nematic cell; quantum-mechanical level anti-crossing; centersymmetric $\left(\chi^{(3)}\right)$ nonlinearity and self-rotation of polarization ellipse; angular momentum conservation; non-center-symmetric $\left(\chi^{(2)}\right)$ nonlinearity and second harmonic generation. This pendulum continues to serve as a source of ideas for new optical devices.
\end{abstract}

Key words: education, optics, anisotropy, liquid crystalline displays, pendulum, adiabatic propagation.

\title{
1. MOTIVATION
}

Many problems in optics are not easily understood by beginners. One of the reasons is that the periods of light wave are about $10^{-14}$ seconds in time and about $10^{-4}$ centimeters in space; therefore this periodic structure can not be resolved by a human eye. Besides that, the human eye is not sensitive to the polarization. A pendulum with two transverse degrees of freedom turned out to be a very useful tool to teach many ideas of oscillations theory, classical mechanics, optics, and even quantum mechanics.

\section{BASIC DEVICE}

The basic device shown in Figure 1. It consists of a rotary platform, one horizontal and two vertical bars, attached to the platform, and a pendulum on a thread. Denote the vertical direction as $z$. Specific feature of the attachement of the thread to the horizontal bar (see Fig. 1) is that the effective length $L_{2}$ for the pendulum oscillations in the frame plane $(x, z)$ is slightly shorter, than the corresponding quantity $L_{1}$ for the oscillations in the perpendicular $(y, z)$ plane. Models with the pendulum length $L<1$ foot are easier to transport. However, they usually show relatively large damping; especially disturbing is that the damping is stronger for one of the polarizations (usually for the $x$ one). 

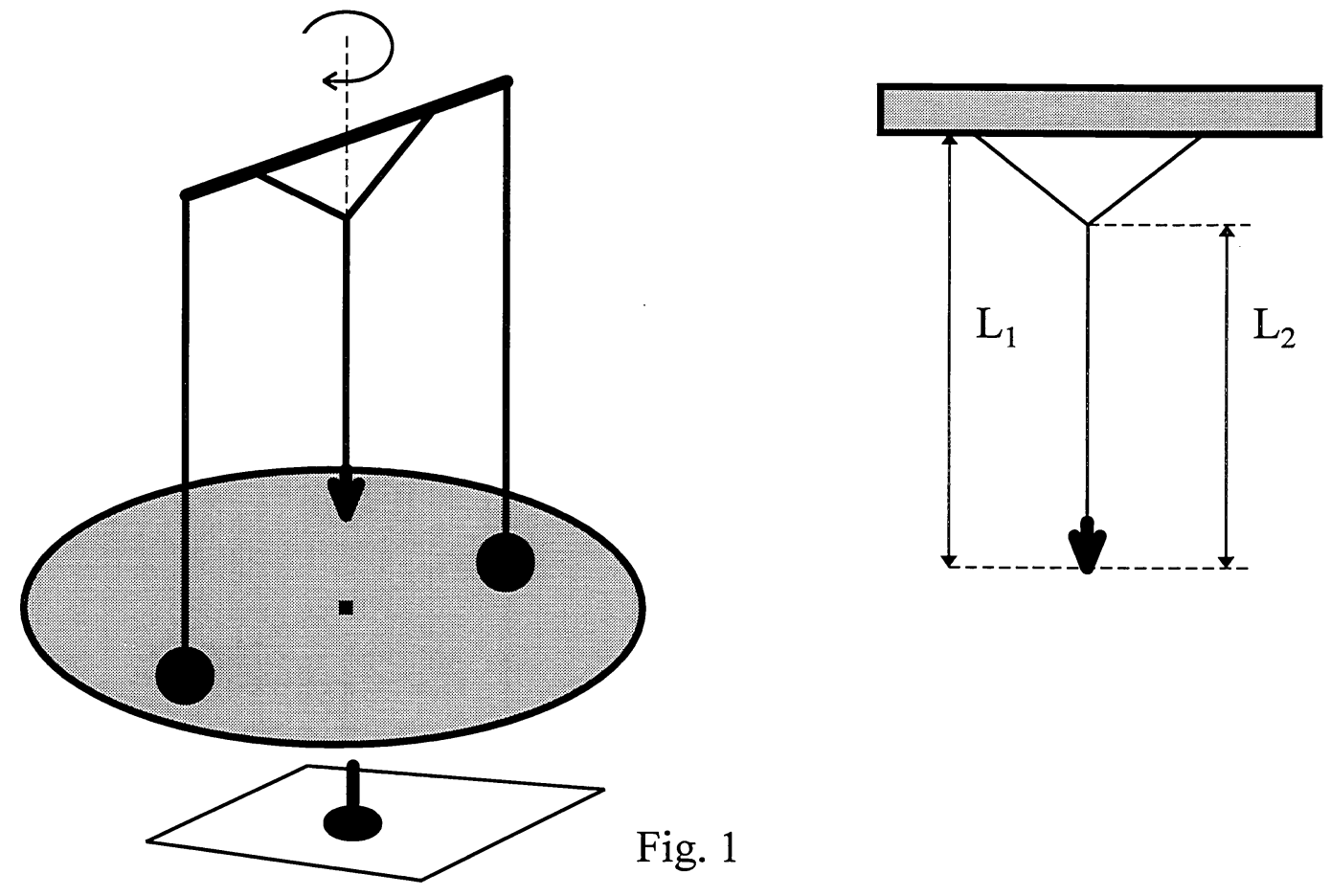

Value of $L$, about 2 feet, gave a very nice performance, with a piece of $1 / 2$-inch thick plywood as a platform, using two rotary elements from boat seats as the rotary stand. Reasonably heavy plumb bob assured both good axial symmetry and low damping. One thread (of three) of a nylon rope was used, to eliminate tension-dependent torque. Very rigid frame connection with the platform was achieved with the use of cast steel plumber's flanges, while the frame was made of $3 / 4$ inch steel plumbing pipes and right angle couplers. The device may be aseembled or disassembled in 5 minutes or less, and may be transported by one person.

If the demonstration does not require rotary platform, the door frame with two nails (about $1 / 2$ foot apart) hammered into the upper bar, is almost ideal due to extreme rigidity, and gives very low damping for a heavy enough plumb bob.

Below we describe the mechanical phenomena to be studied with the use of this pendulum, and their optical analogs. 


\section{EXPERIMENTS WITH THE PENDULUM AND IDEAS TO BE TAUGHT.}

1. Harmonic oscillations of the pendulum,

$$
x=A \cos (\omega t-\varphi)
$$

with small $(A \ll L)$ amplitude allow to introduce the notions of: $\left.{ }^{*}\right) A$, amplitude; $\left.{ }^{*}\right) T=2 \pi \sqrt{L / g}$, period; *) $f=1 / T$, frequency; *) $\omega=2 \pi f$, angular frequency; $\left.{ }^{*}\right) \varphi$, phase.

2. Linearity and superposition. Let $x_{1}(t)=A_{1} \cos (\omega t)$ and $x_{2}(t)=A_{2} \cos (\omega t)$ be the solutions of the (yet unknown) dynamic equations of motion. The hypothesis of linearity, or superposition principle, means that the sum of those solutions is a solution also:

$$
x=A_{1} \cos \omega t+A_{2} \cos \omega t=\left(A_{1}+A_{2}\right) \cos \omega t .
$$

(for small amplitudes, $A \ll L$ ). Hence the easely measured independence of the period $T=2 \pi / \omega$ on the amplitude implies superposition and linearity.

3. Energy: it is proportional to $A^{2}$.

4. Damping is governed by the geometric progression law:

$$
\frac{A^{2}(t+2 \tau)}{A^{2}(t+\tau)}=\frac{A^{2}(t+\tau)}{A^{2}(t)}<1
$$

This is the only law that simultaneously satisfies two conditions. 1) Linearity: if $A(t)$ is a solution, then const $\cdot A(t)$ is a solution as well. 2) Time-shift invariance (stationary system): if $A(t)$ is a solution, then $A(t+\tau)$ is a solution as well.

5. Two dimensions for the oscillations: in $(x, y)$-plane; $z$-component of the displacement is small.

6 . Axial symmetry (isotropy in $x, y$-plane for a one-point hanged pendulum):

$$
\omega_{1}(\text { for } \mathrm{x})=\omega_{2}(\text { for } \mathrm{y})=\omega(\text { for any small } \mathrm{A}) \text {. }
$$

Therefore

$$
\begin{aligned}
& x(t)=x_{0} \cos \left(\omega t-\varphi_{x}\right), \\
& y(t)=y_{0} \cos \left(\omega t-\varphi_{y}\right),
\end{aligned}
$$

and general linear $\left(\varphi_{x}=\varphi_{y}\right)$, elliptical, or circular $\left(x_{0}=y_{0}, \varphi_{x}=\varphi_{y} \pm \pi / 2\right)$ polarization is preserved in time.

7.Anisotropy: $\omega_{1}($ for $\mathrm{x}) \neq \omega_{2}($ for $\mathrm{y})$

$$
\begin{gathered}
x(t)=x_{0} \cos \left(\omega_{1} t\right), \\
y(t)=y_{0} \cos \left(\omega_{1} t+\left(\omega_{2}-\omega_{1}\right) t\right),
\end{gathered}
$$

where $\omega_{1,2}=\sqrt{g / L_{1,2}}$, and $\omega_{2}-\omega_{1}$ is the beats frequency. Since the phase difference $\varphi_{y}-\varphi_{x}=\left(\omega_{2}-\omega_{1}\right) t$ gradually grows in time, one observes gradual transformation of polarization: 
linear at $=+45^{\circ} \rightarrow$ right elliptical at $\approx+45^{\circ} \rightarrow$ right circular $\rightarrow$ right elliptical at $\approx-45^{\circ} \rightarrow$ linear at $-45^{\circ} \rightarrow$ left elliptical at $\approx-45^{\circ} \rightarrow$ left circular $\rightarrow$ left elliptical at $\approx+45^{\circ} \rightarrow$ linear at $+45^{\circ}$.

8. Sharp rotation of the platform during the time interval $\tau \ll\left|\omega_{1}-\omega_{2}\right|^{-1}$ does not change the polarization in the absolute space.

9. Slow rotation of the platform, $\Omega \approx \tau^{-1} \ll\left|\omega_{1}-\omega_{2}\right|$, leads to the adiabatic preservation of the mode type. Explanation requires the ideas of weak excitation of the pendulum by a non-resonant force, and of strong excitation in case of resonance. By the way, blowing from one's mouth is a good way to apply static or resonant external force to the pendulum, with clearly limited modulus of the force. Now, reason for the adiabatic following: each small turn of the platform gives a small excitation of the "alian" mode, but the overall excitation is ineffective, since it is not in resonance, if $\Omega \ll\left|\omega_{1}-\omega_{2}\right|$.

Extra comment: "alian" mode is somewhat "admixed" in the process of rotation, but it is almost totally "outmixed" (cancelled), if the switching of the platform rotation "on" and "off" are smooth enough.

The optical analog of the latter phenomenon is the following. Let a light pulse propagate through a medium with resonant atoms, but in the transparency region. Then during the very process of interaction with the light, the upper atomic state is somewhat "admixed" to the original (ground) state, giving rise to the contribution to the refractive index. However, after the light pulse has gone, the upper state is "outmixed" almost perfectly: no absorbtion in the transparency region.

10. Unusual polarizational properties of symmetric $\left(\chi^{(3)}\right)$ nonlinearity of a point-hanging (isotropic) pendulum.

A) Frequency decreases as

$$
\omega=\omega_{0}\left(1-A^{2} / 16 L^{2}+\cdots\right)
$$

with the increase of the amplitude $A$ of linearly polarized oscillations.

B) Frequency increases as

$$
\omega=\omega_{0} / \sqrt{\cos \theta}=\omega_{0}\left(1+\theta^{2} / 4+\cdots\right)
$$

for the circularly polarized (conical) motion, with the increase of the deflection angle $\theta$.

C) Elliptically polarized motion of finite amplitude 1) preserves the ratio of axes of the ellipse due to conservation of angular momentum,2) preserves the size of the ellipse due to energy conservation, and 3 ) results in a gradual (self-) rotation of the major axis. Corresponding angular velocity $\Omega$ is about $\omega_{0} A^{2} / L^{2}$, and has the same sign, as the rotation of the pendulum inside the ellipse.

11. A system of four coupled differential equations for $x, d x / d t, y, d y / d t$ allows to get chaos and strange attractors. However, the slowly varying complex amplitude approach is insufficient to get chaos. This chaos was observed by us in computer experiments only: one must wait a lot of time, and physical pendulum shows damping long before that.

12. Eigenmodes of bifrequency pendulum at a platform, which rotates with a large, but constant, angular velocity $\Omega$, have the eigenfrequencies

$$
\lambda_{1,2}=\left(\omega_{x}+\omega_{y}\right) / 2 \pm \sqrt{\left(\omega_{x}-\omega_{y}\right)^{2} / 4+\Omega^{2}}
$$


due to the action of the Coriolis force. Level anti-crossing in quantum mechanics thus may be illustrated. Adiabatic change from $\Omega=0$ to $\Omega \gg\left|\omega_{x}-\omega_{y}\right|$ excites counter-rotating circular motion, if one starts with the higher-frequency ( $x$-polarized) eigenmode of a still platform.

13. Non-symmetric (relative to $y \rightarrow-y$ ) nonlinearity of the thread support allows to demonstrate Second Harmonic Generation as a resonant (phase-matched) process. One needs to get $\omega_{x}=2 \omega_{y}$, i. e. $L_{x}=L_{y} / 4$. Fine experimental adjustment of $\omega_{x} / \omega_{y}$ ratio is facilitated by the observation of a stable Lissageoux pattern.

\section{PROPERTIES OF LIGHT TO BE DISCUSSED}

\section{IN PARALLEL WITH THE CORRESPONDING DEMONSTRATIONS OF THE PENDULUM.}

1a. Periodicity in time:

$$
E(t)=E_{0} \cos (\omega t-\varphi)
$$

Since $\omega / 2 \pi$ is about $10^{14} \mathrm{~Hz}$, that is not where the analogy will be mostly applied.

1b. Propagation of light in space:

$$
E(z, t)=E_{0} \cos \left(\omega\left(t-\frac{z n}{c}\right)-\varphi\right) .
$$

Evolution of light wave along $z$-direction will be concidered as analogous to the time evolution of the pendulum oscillations. Additional notions: $\left.{ }^{*}\right) n$, refractive index; $\left.{ }^{*}\right) c / n$, phase velocity of the wave; ${ }^{*}$ ) $\lambda=\lambda_{\mathrm{vac}} / n$, spatial period in the medium; $\left.{ }^{*}\right) \lambda_{\mathrm{vac}}=2 \pi c / \omega$, wavelength in the vacuum.

2. Independence of refractive index (and hence, of spatial period $\lambda=n^{-1} \lambda_{\text {vac }}$ ) on the amplitude $E$; linearity and superposition:

$$
E=E_{1} \cos (\omega t)+E_{2} \cos (\omega t)=\left(E_{1}+E_{2}\right) \cos (\omega t)
$$

(for small amplitudes, $E \ll E_{\text {at }}, E_{\text {at }} \approx 10^{11}$ Volt/meter).

3. Power is proportional to $E^{2}$.

4. Exponential law for the attenuation due to absorption:

$$
E_{0}^{2}(z)=\text { const } \cdot \exp (-\alpha z)
$$

Non-monochromatic light may contain spectral components $\omega_{i}$ with different values of absorption coefficient $\alpha\left(\omega_{i}\right)$. That results in the deviations from simple exponential law for the total intensity. Dichroism also leads to such deviations.

5. Polarization of light: the vector $\mathbf{E}$ is in the $(x, y)$-plane, perpendicular to the propagation direction $z$. This analogy with the pendulum is rather superficial, but it is extremely visual !

6 . If the medium is isotropic (at least, in $(x, y)$-plane), then

$$
n_{1}(\text { for } \mathrm{x})=n_{2}(\text { for } \mathrm{y})=n(\text { for any direction of.E })
$$


and

$$
\begin{aligned}
& E_{x}(z)=E_{0 x} \cdot \cos \left(\frac{\omega n}{c} z+\varphi_{x}-\omega t\right), \\
& E_{y}(z)=E_{0 y} \cdot \cos \left(\frac{\omega n}{c} z+\varphi_{y}-\omega t\right) .
\end{aligned}
$$

Any polarization is preserved during the propagation: either linear $\left(\varphi_{x}=\varphi_{y}\right)$, or elliptical, or right circular $\left(E_{0 x}=E_{0 y}, \varphi_{x}=\varphi_{y}-\pi / 2\right)$, or left circular $\left(E_{0 x}=E_{0 y}, \varphi_{x}=\varphi_{y}+\pi / 2\right)$.

7. Teaching three aspects of anisotropy and birefringence in optics.

7a. Group velocities are not collinear for two polarizations. Parallel slab of about $1 \mathrm{~cm}$ thick of a calcite crystal produces visible doubling of image, with transverse shift $\Delta x \approx \eta \cdot 1 \mathrm{~cm} \approx 0.1 \mathrm{~cm}$, since the angle between the group velocities of the o- and e-waves is $\eta \approx \Delta n / n \approx 0.1$.

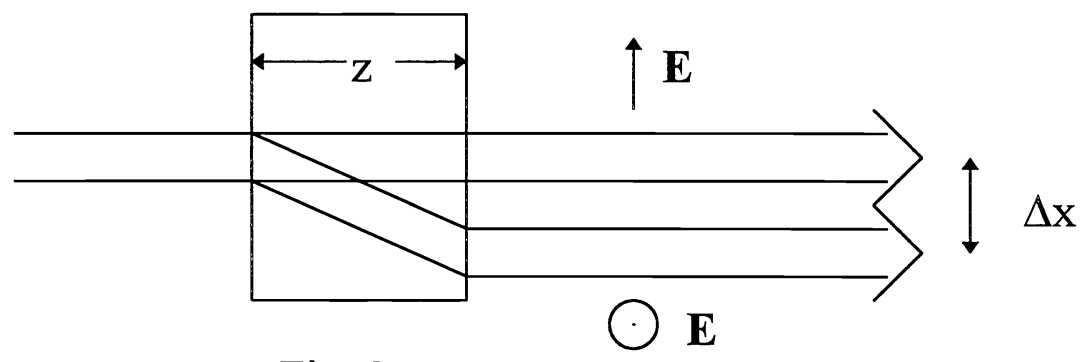

Fig. 2

$7 \mathrm{~b}$. The difference in refractive forces for two polarizations leads to the different deflection of a ray by a prizm: $\Delta x \approx 5 \mathrm{~cm}$ for the prizm angle $\beta$ about $30^{\circ}, \Delta n / n \approx 0.1$ and $L$ about $1 \mathrm{~m}$.

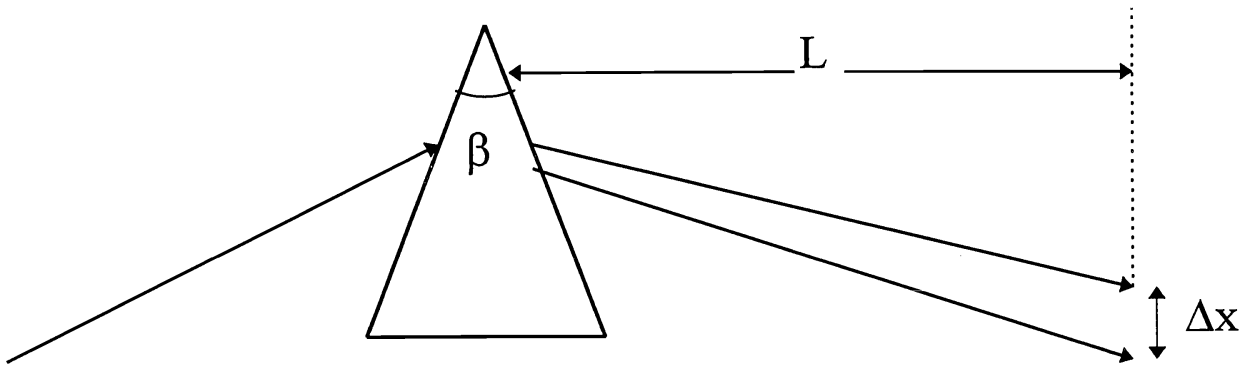

Fig. 3

7c. Change of polarization due to the propagation - the strongest effect:

$$
\begin{gathered}
E_{x}(z)=E_{0 x} \cdot \cos \left(\frac{\omega n_{1}}{c} z-\omega t\right), \\
E_{y}(z)=E_{0 y} \cdot \cos \left(\frac{\omega n_{1}}{c} z-\omega t+\frac{\omega}{c}\left(n_{1}-n_{2}\right) z\right) .
\end{gathered}
$$

Simple estimations:

$$
z=10^{-2} \mathrm{~cm}, n_{1}-n_{2}=10^{-4}, \lambda_{\text {vac }}=0.6 \text { microns, } \varphi_{x}-\varphi_{y}=10 \text { radians } !
$$


Colors of a plastic piece between crossed polarizers are explained by this, the strongest, manifestation of birefringence. Just this phenomenon is modelled by a bifrequency pendulum on a still frame.

8. Sharp spatial rotation of the optical axis of a liquid crystal, at a distance $L \ll \lambda /\left|n_{1}-n_{2}\right|$, does not change the polarization in the absolute space (in analogy with the pendulum).

9. For the slow spatial rotation of the optical axis of liquid crystal, at a distance $L \gg \lambda /\left|n_{1}-n_{2}\right|$, the polarization follows adiabatically the optical axis.

The principle of work of the Liquid Crystalline Display (LCD) is based on the switching between propagation in the conditions of sharp versus adiabatic changes.
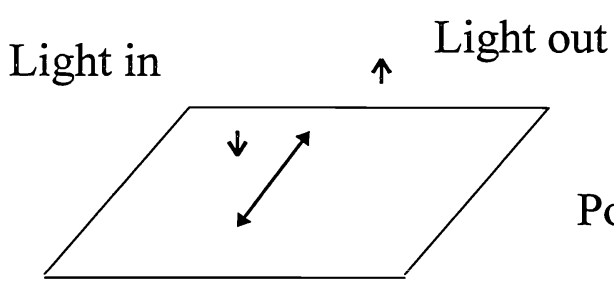

Polarizer

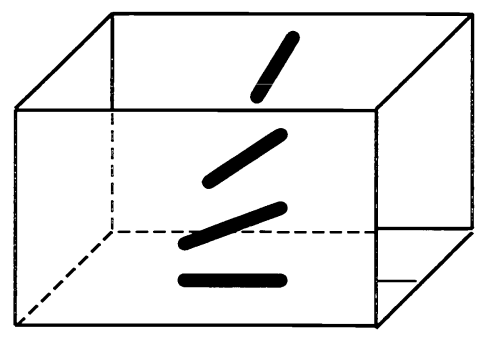

Twisted nematic LC

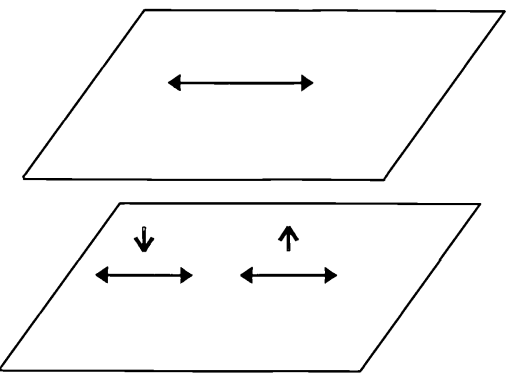

Fig. 4a

Analyzer

Diffusive reflector

\section{Light in No light out}
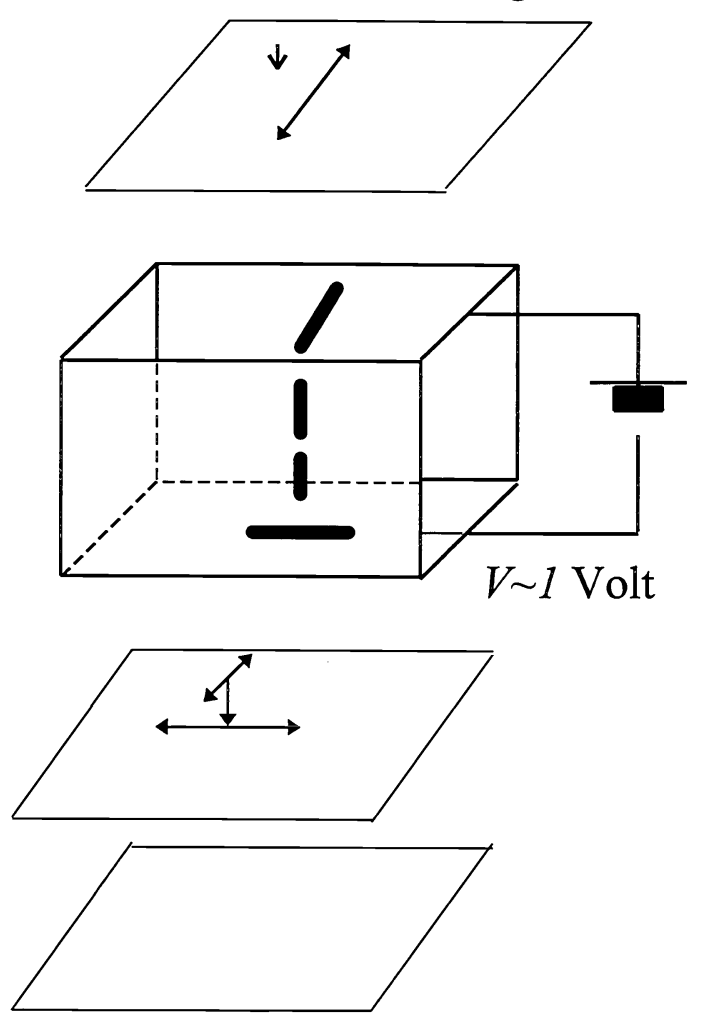

Fig. $4 b$ 
No voltage on LC layer $\rightarrow$ adiabatic following of polarization $\rightarrow$ light is transmitted forth and back $\rightarrow$ white field of the LCD. All the display is white, if the battery is out.

Applied voltage changes the LC orientation very sharply $\rightarrow L$ and $n_{1}-n_{2}$ are diminished $\rightarrow$ polarization is preserved in absolute space $\rightarrow$ light is blocked by analyser $\rightarrow$ black stripe on LCD. All the display is black, if LC leaked out.

10. Self-rotation of polarization ellipse in an optically nonlinear $\left(\chi^{(3)}\right)$ medium is analogous to the effect for the pendulum.

11. Adiabatic passage of an atom from the ground state into the excited state in a resonant field with sweeping frequency is based on the level anti-crossing.

12. Chaos may be observed for optical propagation.

13. Second Harmonic Generation is one of the most popular processes in modern technology of laser light.

\section{CONCLUSION}

OPTICS AND MECHANCS ARE SIMILAR:

IT IS FUN TO TEACH THEM ! 